\title{
Die Stellung der Privaten Krankenversicherung im System der Sozialen Sicherung in der Bundesrepublik Deutschland
}

\author{
von Hans Georg Timmer*
}

\begin{abstract}
Das deutsche Krankenversicherungssystem ist gekennzeichnet durch das Nebeneinander zweier historisch gewachsener Teilsysteme, der gesetzlichen Krankenversicherung und der privaten Krankenversicherung. Der privaten Krankenversicherung kommt im Rahmen dieses Gesamtsystems eine eigenständige und tragende Bedeutung zu. Im Unterschied zu vielen europäischen Nachbarländern, in denen die Sozialversicherung oder ein nationaler Gesundheitsdienst das völlig dominierende System und die private Krankenversicherung auf eine Ergänzungsfunktion beschränkt ist, ist die deutsche private Krankenversicherung einer der beiden maßgeblichen Träger der Absicherung im Krankheitsfall, und zwar für bestimmte Bevölkerungsgruppen. Darüber hinaus nimmt sie aber auch ergänzende Funktion zur Sozialversicherung wahr.
\end{abstract}

Dies kann man anhand einiger Zahlen kurz verdeutlichen : Im Jahre 1986 waren rd. $89 \%$ der bundesdeutschen Bevölkerung im Rahmen der gesetzlichen Krankenversicherung versichert, knapp $9 \%$ hatten eine private Vollversicherung, d. h. sie waren ausschließlich privat versichert. Weitere rd. 7\% der Bevölkerung, die im Rahmen der gesetzlichen Krankenversicherung abgesichert waren, hatten eine private Zusatzversicherung ( $z$. B. für Krankenhausunterbringung).

Insgesamt waren somit über $16 \%$ der Bevölkerung in irgendeiner Form privat versichert. Der potentielle Wettbewerbsbereich von gesetzlicher und privater Krankenversicherung umfaßt schätzungsweise $25 \%$ der Bevölkerung; hierbei handelt es sich um Personengruppen, die zwischen beiden Trägersystemen der Krankenversicherung wählen können, d. h. um Personen, die

- entweder grundsätzlich versicherungspflichtig in der gesetzlichen Krankenversicherung sind, aber ein Recht auf Befreiung von dieser Versicherungspflicht haben,

- oder grundsätzlich nicht versicherungspflichtig in der gesetzlichen Krankenversicherung sind, aber ein Recht auf Beitritt oder Weiterversicherung haben.

* Chairman of the Board of Management, Deutsche Krankenversicherung AG Köln; Paper presented at the 14th General Assembly of the Geneva Association, Berlin, June 15, 1987. 
Soviel zu den quantitativen Aspekten. Wie lassen sich nun die Stellung der privaten Krankenversicherung im Gesamtsystem und ihre besonderen Existenzbedingungen näher charakterisieren? In diesem Zusammenhang kommt den unterschiedlichen Ordnungsprinzipien und Funktionsmerkmalen von gesetzlicher und privater Krankenversicherung eine grundlegende Bedeutung zu.

Die Ausgestaltung des Systems der sozialen Sicherung in Deutschland berücksichtigt in seinen beiden Teilen private Krankenversicherung und gesetzliche Krankenversicherung die für unsere Gesellschafts- und Wirtschaftsordnung maßgeblichen Ordnungsprinzipien : das Gundrecht jedes einzelnen auf freie Entfaltung der Persönlichkeit einerseits und das Sozialstaatsprinzip andererseits. Damit bildet das Krankenversicherungssystem das Spannungsfeld $a b$, das im gesellschaftlichen Zusammenleben zwischen dem einzelnen und der Gesellschaft, zwischen persönlicher Entscheidungsfreiheit und sozialer Bindung besteht. Das Sozialstaatsprinzip berechtigt und verpflichtet den Staat, sozial gestaltend tätig zu werden. Aus dem Rechtsstaatsprinzip, das der persönlichen Freiheit und Entfaltungsmöglichkeit des einzelnen den höchsten Stellenwert einräumt, folgt auf der anderen Seite, daß die Einschränkung der Freiheit des einzelnen nur aus übergeordneten Gründen erfolgen darf und auf den notwendigen Umfang beschränkt bleiben muß. Das bedeutet, es kann weder eine ausschließlich individuelle und eigenverantwortliche Krankheitsvorsorge geben, noch darf eine Deckung des Krankheitsrisikos ausschließlich in staatlicher Verantwortung erfolgen.

Den Maßstab für die Berücksichtigung und den Ausgleich der beiden Prinzipien im skizzierten Spannungsfeld bildet das Subsidiaritätsprinzip. In der Solidargemeinschaft der gesetzlichen Krankenversicherung ist demnach die Gruppe der Schutzbedürftigen zusammenzufassen, denen aus eigener - finanzieller - Kraft die selbstverantwortliche Absicherung gesundheitlicher Risiken nicht möglich ist. Der Definition des Begriffs "Schutzbedürftigkeit" ist hierbei besondere Sorgfalt zu widmen. Nicht Schutzbedürftigen hingegen muß die eigenverantwortliche und risikogerechte Absicherung im Rahmen der PKV oder sonstiger privater Schutzgemeinschaften nicht nur möglich sein, sondern auch zugemutet werden.

Ich möchte an dieser Stelle nicht näher darauf eingehen, inwieweit die in Deutschland bestehende konkrete Abgrenzung der beiden Teilsysteme diese grundlegenden Prinzipien in einer ausgewogenen Form berücksichtigt bzw. in wieweit hier aus meiner Sicht Akzentverschiebungen diskutiert werden sollten. Es sei mir lediglich am Rande der Hinweis gestattet, daß nach meinem Verständnis von einer subsidiären Funktion des Staates kaum $90 \%$ der Bürger eines wohlhabenden Landes "schutzbedürftig" sein können. Entscheidend kommt es mir jedoch hier auf die grundsätzliche Geltung der beiden Prinzipien an. Sie finden ihren Niederschlag in der konkreten Ausgestaltung und Funktionsweise der beiden Versicherungssysteme.

Die privaten Krankenversicherungen sind privatrechtliche Einrichtungen. Der Beitritt erfolgt grundsätzlich auf freiwilliger Basis. Vertragsabschluß und Gestaltung unterliegen im Rahmen bestimmter gesetzlicher Einschränkungen der freien Vereinbarung der Beteiligten. Demgegenüber sind die gesetzlichen Krankenversicherungen öffentlich-rechtliche Einrichtungen; der Beitritt beruht in wesentlichen Teilen auf der Versicherungspflicht, Beitrags- und Leistungsgestaltung sind durch die Sozialgesetzgebung im Rahmen der sogenannten "Reichsversicherungsordnung" weitgehend festgelegt. 
Finanzierung, Beitrags- und Leistungsbemessung erfolgen in beiden Systemen nach verschiedenen Prinzipien : Grundlage der Beitragsbemessung in der privaten Krankenversicherung bildet das Äquivalenzprinzip, d. h. jede versicherte Person zahlt wagnisgerechte Beiträge unter Berücksichtigung von vereinbarter Leistungshöhe, Geschlecht und Alter. Demgegenüber strebt die gesetzliche Krankenversicherung nicht nur einen versicherungsmäßigen Risikoausgleich an, sondern auch einen sozialen Ausgleich. Die einzelnen Beiträge werden ohne Rücksicht auf die bei der privaten Krankenversicherung zu beachtenden Risikofaktoren in Abhängigkeit vom Einkommen des Mitglieds bemessen, und zwar unabhängig davon, wie viele mitversicherte Familienangehörige des Mitglieds Leistungen im Krankheitsfall erhalten.

Im Hinblick auf das Leistungsangebot stehen sich bei den beiden Krankenversicherungsystemen auf der einen Seite normierte Standardleistungen der gesetzlichen Krankenversicherung und auf der anderen Seite individuelle Leistungsgestaltung der privaten Krankenversicherung gegenüber. Die gesetzliche Krankenversicherung kann gemäß ihrem Auftrag lediglich einen Standardversicherungsschutz anbieten, der zwar ausreichend und zweckmäßig sein muß, das $\mathrm{Maß}$ des Notwendigen jedoch nicht überschreiten darf.

Die Wettbewerbschance der privaten Krankenversicherung liegt dagegen in dem Angebot vielfältiger und flexibler Leistungsvarianten, wobei die individuellen Bedürfnisse und Wünsche der Kunden das Maß aller Dinge sind. Der Versicherte kann aus den variablen Möglichkeiten ein Leistungsangebot auswählen, das seinen Bedürfnissen und finanziellen Möglichkeiten entspricht. So gehören zur Angebotspalette der privaten Krankenversicherung sowohl Mehrleistungen gegenüber dem gesetzlichen Versicherungsschutz als auch Vereinbarungen über Minderleistungen. Zu den Mehrleistungen zählen beispielsweise bessere Unterbringung im Krankenhaus und die Inanspruchnahme von Chefarztbehandlung, die Sicherung des vollen Einkommensausfalls bei Arbeitsunfähigkeit sowie Krankenhaustagegeld. Vereinbarungen von Minderleistungen sind die zahlreichen Varianten von Selbstbeteiligungen, die mit erheblichen Beitragsreduzierungen verbunden sind und einen Anreiz zum Gesundbleiben und zur maßvollen Inanspruchnahme medizinischer Leistungen darstellen. Fragen der Gestaltung des Leistungsangebots und insbesondere auch Überlegungen im Hinblick auf neue Angebots- und Finanzierungsformen werden aus meiner Sicht eine der Hauptherausforderungen der Zukunft für die private Krankenversicherung sein.

In Zeiten ständig steigender Kosten im Gesundheitswesen und damit auch steigender Krankenversicherungsbeiträge kommt der Höhe der Beiträge und dem Beitragswettbewerb eine entscheidende Bedeutung zu. Der Wettbewerb vollzieht sich auf der Grundlage der bereits skizzierten unterschiedlichen Finanzierungsverfahren, dem Solidarprinzip bei der gesetzlichen Krankenversicherung, das in einem Umlageverfahren bei der Beitragserhebung seinen Ausdruck findet, und dem Äquivalenzprinzip bei der privaten Krankenversicherung, bei dem risikogerechte Beiträge une eine Alterungsreserve wesentliche Gestaltungsmerkmale sind. Aus den systembedingten Unterschieden in der Kalkulation ergibt sich eine unterschiedliche Attraktivität der privaten Krankenversicherung für bestimmte Personenkreise und damit eine Einschränkung des faktischen Wettbewerbsbereichs. So ist das private Angebot eher uninteressant für ältere Personen und größere Familien mit nur 
einem Verdiener, während sich tendenziell jüngere und alleinstehende Personen, ZweiVerdiener-Haushalte und kleinere Familien in der privaten Krankenversicherung günstiger versichern können. Im Hinblick auf die letztgenannten Gruppen kommt der Einrichtung der Beitragsrückerstattung, die die private Krankenversicherung den Versicherten gewährt, die ein oder mehrere Jahre keine Leistungen in Anspruch genommen haben, als zusätzlichem Wettbewerbsfaktor eine besondere Bedeutung zu.

Wenn man die Stellung der privaten Krankenversicherung im System der sozialen Sicherung und ihre besonderen Existenzbedingungen betrachtet, so dürfen aus meiner Sicht die erheblichen Wettbewerbsnachteile nicht unerwähnt bleiben, denen sie im Verhältnis zur gesetzlichen Krankenversicherung unterliegt. Ich kann sie an dieser Stelle nur kurz aufzählen :

- Die private Krankenversicherung verfügt erstens über keine Versicherungspflicht, sondern muß alle ihre Versicherten werben.

- Sie hat zweitens - anders als die gesetzliche Krankenversicherung - keine steuerlichen Privilegien in bezug auf Körperschaft-, Gewerbe- und Vermögensteuer.

- Sie kann drittens nicht wie die gesetzliche Krankenversicherung einen Teil ihrer Betriebs- und Verwaltungskosten auf Dritte abwälzen.

- Und sie muß viertens die Leistungen im Gesundheitswesen meist teurer bezahlen als die gesetzliche Krankenversicherung; dies gilt insbesondere für Arztleistungen, aber auch z. B. für Medikamente.

In der privaten Krankenversicherung haben wir notgedrungen gelernt, mit diesen Nachteilen zu leben und trotz dieser Nachteile erfolgreich im Wettbewerb zu bestehen. Gerade mit Blick auf die zukünftige Entwicklung des deutschen Krankenversicherungswesens und die anstehende Reform - Themen des morgigen Tages, denen ich hier nicht vorgreifen möchte - ist es jedoch existenziell wichtig für uns, daß sich die Wettbewerbsbedingungen zumindest nicht weiter verschlechtern. Natürlich ist es unser stetiges Ziel, die Bedingungen tendenziell zu verbessern.

Im Zusammenhang mit den Existenzbedingungen der privaten Krankenversicherung kann die Problematik einer gravierenden Kostenentwicklung im Gesundheitswesen nicht unerwähnt bleiben. Sie gehört - in wechselndem Ausmaß - seit rund 20 Jahren zu den wesentlichen Rahmenbedingungen; allerdings stellt sich die Entwicklung insgesamt günstiger dar als bei der gesetzlichen Krankenversicherung. Die Bewältigung der Kostenproblematik ist sicher eine der Hauptaufgaben der nächsten Jahre und Jahrzehnte, eine Aufgabe, die sich dem gesamten Gesundheits- und Krankenversicherungswesen stellt. Angesichts der sich abzeichnenden Expansionsfaktoren - der zunehmenden Überalterung der Bevölkerung, der Zunahme chronischer Krankheiten, der Weiterentwicklung des medizinischen Fortschritts, der Ärzteschwemme - wird es dringend notwendig sein, nach Wegen zu suchen, die Qualität und Bezahlbarkeit des deutschen Gesundheitswesens sicherstellen.

Abschließend noch einige Bemerkungen zur speziellen Bedeutung der privaten Krankenversicherung als unverzichtbarer Mitträger der sozialen Sicherung in Deutschland: Die private Krankenversicherung gibt durch die Eröffnung von Wahlfreiheit und Handlungsspielräumen der Eigenverantwortung den ihr gebührenden Raum. Sie erfüllt damit in 
unserer freiheitlichen und pluralistischen Gesellschaft, die den Menschentyp des mündigen Bürgers postuliert, eine wesentliche gesellschaftspolitische Funktion. Die private Krankenversicherung gewährleistet darüber hinaus die Existenz marktwirtschaftlicher Elemente im Gesundheits- und Krankenversicherungswesen. Ihre Existenz und ihr Wettbewerb zur gesetzlichen Krankenversicherung tragen in erheblichem Umfang zur Förderung von Leistungsfähigkeit, Preiswürdigkeit und Versichertennähe des Gesamtsystems bei. Letztlich ist der Wettbewerb ein stetiger Anreiz zur Steigerung der Effizienz und zur Entwicklung fortschrittlicher Ideen und Innovationen. Gerade auf dem Gebiet der Innovationen sehe ich eine besondere Aufgabe und Chance für die private Krankenversicherung, die hier systembedingt über mehr Spielraum verfügt. Es wird nicht zuletzt entscheidend vom Ausmaß unserer Innovationsfähigkeit und unserer Flexibilität abhängen, ob es uns gelingt, die Herausforderungen der Zukunft positiv zu bewältigen und damit einen Beitrag zur Stabilisierung unseres bewährten sozialen Sicherungssystems zu leisten. 